\title{
New method to extract information of near-threshold resonances: Uniformized Mittag-Leffler expansion of Green's function and $T$ matrix
}

\author{
Wren Yamada $\odot^{1,2, *}$ and Osamu Morimatsu $\oplus^{1,2,3, \dagger}$ \\ ${ }^{1}$ Department of Physics, Faculty of Science, University of Tokyo, 7-3-1 Hongo Bunkyo-ku, Tokyo 113-0033, Japan \\ ${ }^{2}$ Theory Center, Institute of Particle and Nuclear Studies (IPNS), High Energy Accelerator Research Organization (KEK), \\ 1-1 Oho, Tsukuba, Ibaraki 205-0801, Japan \\ ${ }^{3}$ Department of Particle and Nuclear Studies, Graduate University for Advanced Studies (SOKENDAI), \\ 1-1 Oho, Tsukuba, Ibaraki 305-0801, Japan
}

(Received 25 May 2020; accepted 19 October 2020; published 5 November 2020)

\begin{abstract}
We propose a new, model-independent approach that appropriately incorporates the resonant and threshold behaviors. We show that when choosing the appropriate variable, the complete Green's function and the $T$ matrix can be expressed as single-valued functions (uniformization) in the form of a simple series explicitly written by the bound and resonant poles: the uniformized Mittag-Leffler expansion. The poles' symmetries, arising from the unitarity of the $S$ matrix, impose the series to obey the proper threshold behaviors. We then demonstrate this method in a model case of a double-channel meson-baryon scattering, with channels, $\bar{K} N(I=0)$, and $\pi \Sigma(I=$ 0 ), by fitting the numerically calculated $T$ matrix with the simple series and comparing the fitted results to the exact results.
\end{abstract}

DOI: 10.1103/PhysRevC.102.055201

\section{INTRODUCTION}

Resonances and threshold behaviors of hadron scatterings are characteristic nonperturbative phenomena in strong interaction physics. From a mathematical perspective, resonances and hadron scattering processes, threshold behaviors, in particular, correspond to poles and branch points of an analytic function, the $S$ matrix. Formally, a resonance is defined by the pole of the scattering amplitude, $\mathcal{A}$, as a Breit-Wigner form [1],

$$
\mathcal{A}(\sqrt{s}) \sim \frac{\Gamma_{R}}{\sqrt{s}-M_{R}-i \frac{\Gamma_{R}}{2}},
$$

or as a relativistic Breit-Wigner form (e.g., Ref. [2]),

$$
\mathcal{A}(s) \sim \frac{M_{R} \Gamma_{R}}{s-M_{R}^{2}-i M_{R} \Gamma_{R}},
$$

where $s$ is the center-of-mass energy squared, and $M_{R}$ and $\Gamma_{R}$ are the mass and the width of the resonance, respectively. These formulas describe observables well if the observed center-of-mass energy, $\sqrt{s}$, is close to the pole mass, $M_{R}$, and is sufficiently distant from the thresholds.

\footnotetext{
*wren-phys@g.ecc.u-tokyo.ac.jp

†osamu.morimatsu@kek.jp
}

Published by the American Physical Society under the terms of the Creative Commons Attribution 4.0 International license. Further distribution of this work must maintain attribution to the author(s) and the published article's title, journal citation, and DOI. Funded by $S C O A P^{3}$.
It is also well known that in the vicinity of the threshold the imaginary part of the scattering amplitude behaves as

$$
\operatorname{Im} \mathcal{A}(\sqrt{s})= \begin{cases}0 & \left(\sqrt{s}<\varepsilon_{1}\right), \\ a k & \left(\sqrt{s}>\varepsilon_{1}\right)\end{cases}
$$

at the lowest threshold and

$$
\operatorname{Im} \mathcal{A}(\sqrt{s})= \begin{cases}c+\alpha \kappa & \left(\sqrt{s}<\varepsilon_{i}\right), \\ c+a k & \left(\sqrt{s}>\varepsilon_{i}\right)\end{cases}
$$

at higher thresholds [3], where $\varepsilon_{i}$ is the threshold energy, $k$ is the momentum in the center-of-mass frame, $\kappa$ is defined by $k=i \kappa$, and $c, a$ and $\alpha$ are real constants. The interrelationship between the resonances and the threshold behaviors create prosperous and sophisticated grounds on hadron physics (e.g., Ref. [4]). One typical example is the existence of exotic hadrons (e.g., Ref. [5]).

Neither the original Breit-Wigner form nor the relativistic Breit-Wigner form incorporates the proper threshold behaviors, making it challenging to extract information on near-threshold resonances from experimental data. Some phenomenological attempts have been made to formulate scattering amplitudes such as Ref. [6], which modifies the Breit-Wigner form to incorporate both resonance and threshold behaviors:

$$
\mathcal{A}(s) \sim \frac{M_{R} \sqrt{\Gamma_{1} \Gamma_{2}}}{s-M_{R}^{2}-i M_{R}\left(\Gamma_{1}+\Gamma_{2}\right)},
$$

where $\Gamma_{i}=g_{i} k_{i}, k_{i}$ is the momentum in the center-of-mass frame, and $g_{i}$ may be considered as the coupling constant squared for the resonance. In Ref. [6], the imaginary part of the denominator of Eq. (5) has an opposite sign relative to the real part. Considering the symmetries of the poles (symmetric 


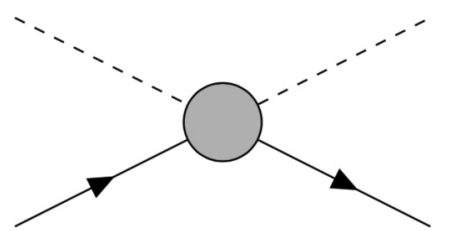

FIG. 1. Two-body (meson-baryon) scattering process. The circle represents the two-body $T$ matrix.

about the positive real axis of the $s$ plane), we unified the notation to Eqs. (3) and (4).

These attempts, however, are far from satisfactory from a theoretical point of view. In this paper, we propose a novel approach that naturally and perfectly incorporates both resonance and threshold behaviors in a theoretically sound fashion based on the analyticity and the unitarity of the $S$ matrix, particularly in coupled channels.

This project's eventual goal is to extract information on near-threshold resonances by applying our method to actual experimental data. In this paper, however, we apply our method to the results of a numerically calculated model theory. Since we can precisely calculate the pole energies and residues within the model theory, we can test our method by comparing the fitted results to the exact results. The results applying our method to actual experimental data will be shown in a subsequent paper.

The study of resonances in coupled channels has a long history [7]. In particular, the methods of the $K$ matrix [8] and the dispersion relation [9] have been widely used and worked out. Our method can be regarded as a variation of the dispersion relation. It, however, differs in representing the $S$ matrix (and the Green's function) as a single-valued function of a simple variable, called uniformization, which enables us to incorporate the analyticity and unitarity of the $S$ matrix (the Green's function), particularly in coupled channels in a simple fashion.

\section{UNIFORMIZED MITTAG-LEFFLER EXPANSION OF THE GREEN'S FUNCTION AND $T$ MATRIX}

To extract information on resonances from experimental data, we must link the experimental observables to analytic functions, such as the $T$ matrix or the Green's function. Here, we briefly review their relations and explain our notations used in the present paper, having in mind the resonances in the meson-baryon system. The most typical observable to explore resonances in the meson-baryon system is the meson-baryon scattering cross section, $\sigma$, as shown in Fig. 1. $\sigma$ is related to the imaginary part of the $T$ matrix via the optical theorem as

$$
\sigma \propto \operatorname{Im} \mathcal{T} .
$$

We can also think of other observables such as the mesonbaryon invariant or missing-mass distribution, which are obtained by selecting the meson-baryon channel of interest as a part of the final states of some reaction experiment as shown in Fig. 2. The amplitude of the distribution, $\mathcal{N}$, is proportional to the imaginary part of the Green's function as [10-12]

$$
\mathcal{N} \propto \operatorname{Im} \mathcal{G} .
$$

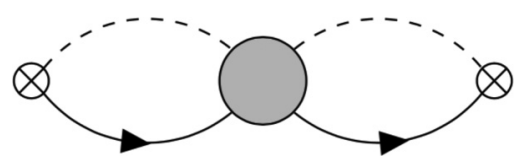

FIG. 2. Diagram for the invariant or missing-mass distribution. The circles with X's represent the process which creates (annihilates) the meson-baryon channel.

Since the full Green's function, $\mathcal{G}$, the free Green's function, $\mathcal{G}_{0}$, and the $T$ matrix, $T$, are related to each other by the relation

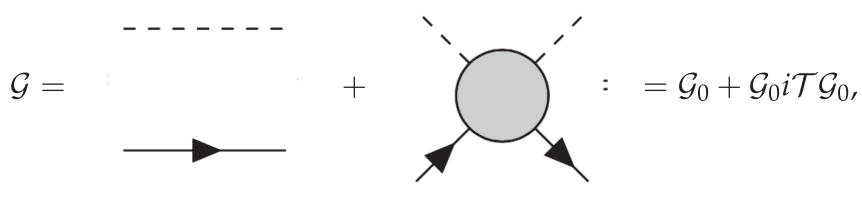

the full Green's function, $\mathcal{G}$, should have the same analytic structure as the $T$ matrix. The following argument is based on the analytic structure of Riemann surfaces and can be applied to either case. For our convenience, we use the Green's function in the following discussion.

We start from the spectral representation of the Green's function. The Green's function can be written as the sum of contributions of the bound states, $\left|\phi_{B}\right\rangle\left(s=s_{B}\right)$, and the continuum, $\left|\phi_{C}\right\rangle\left(s=s_{C}\right)$, as

$$
\mathcal{G}(s)=\sum_{B} \frac{\left|\phi_{B}\right\rangle\left\langle\phi_{B}\right|}{s-s_{B}}+\int_{s_{\mathrm{th}}}^{\infty} d s_{C} \frac{\left|\phi_{C}\right\rangle\left\langle\phi_{C}\right|}{s-s_{C}} .
$$

This expansion corresponds to the contour shown in Fig. 3, which detours the branch cuts that run from each threshold to infinity on the complex $s$ plane.

In general the Green's function also has resonant poles, which are located on the unphysical sheets of the complex $s$ plane. Since the Green's function on the unphysical sheets is just an analytic extension of the Green's function on the physical sheet, the contribution from a pole on an unphysical sheet cannot be written in the same manner as the contribution

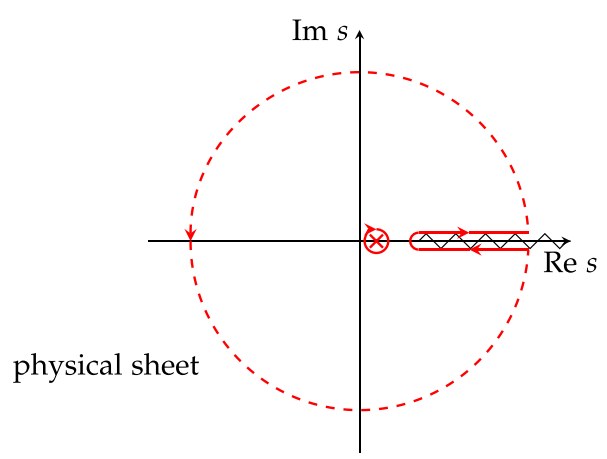

FIG. 3. The contour corresponding to the spectral representation, Eq. (9). The contour detours the branch cuts that run along the real axis. 
from the physical sheet as

$$
\mathcal{G}(s) \neq \sum_{B} \frac{\left|\phi_{B}\right\rangle\left\langle\phi_{B}\right|}{s-s_{B}}+\sum_{R} \frac{\left|\phi_{R}\right\rangle\left\langle\tilde{\phi}_{R}\right|}{s-s_{R}}+\ldots,
$$

where $s_{R}$ are the pole positions on the unphysical sheets of the complex $s$ plane and $\left|\phi_{R}\right\rangle$ and $\left|\tilde{\phi}_{R}\right\rangle$ are the biorthogonal state vectors [13]. The information about resonant poles, such as complex energies or residues, are only implicitly encoded in the continuum contribution in Eq. (9). To decode the information about resonant poles, one must consider a different variable that unfolds the Riemann sheets. This process of unfolding the Riemann surface is called uniformization [3,14]. Uniformization is essential to treat the bound-state poles and resonant poles in the same manner.

Before we discuss uniformization in detail, we note an important property regarding the pole positions and residues of the $S$ matrix. The $S$ matrix satisfies the condition

$$
\mathcal{S}^{*}\left(\left\{-k^{*}\right\}\right)=\mathcal{S}(\{k\}),
$$

where $\{k\}$ represents the set of channel momenta, that is, $\{k\}=k$ in single-channel systems and $\{k\}=k_{1}, k_{2}$ in doublechannel systems. From Eq. (11), the poles of the $S$ matrix (and the Green's function) are symmetric with respect to the imaginary axis. Now consider that the Green's function has a pole at $\left\{k_{0}\right\}$ with a residue $c_{0}$. The symmetry properties imply that there is a pole at $\{k\}=\left\{-k_{0}^{*}\right\}$. By keeping in mind Eq. (11) and the orientation of a contour around $\{k\}=\left\{-k_{0}^{*}\right\}$, it can be shown that the residue at $\{k\}=\left\{-k_{0}^{*}\right\}$ is $-c_{0}^{*}$. To summarize, the poles of the Green's function form a symmetric pair about the imaginary axis of $k$, and the residues of these symmetric poles are related by the complex conjugate of their counterparts.

Now let us move on to the details of uniformization. The appropriate kinetic variable to uniformize the Riemann surface depends on the number of channels considered. For single-channel systems, we define a variable $q$ by

$$
q=\sqrt{s-\varepsilon^{2}}=\sqrt{\frac{\varepsilon}{\mu}} k+O\left(k^{3}\right),
$$

where $\varepsilon$ is the threshold energy and $\mu$ is the reduced mass. $q$ is proportional to the momentum $k$, at the threshold. The spectral representation (Fig. 3) corresponds to the contour in Fig. 4(a). To explicitly write down the contributions from the resonant poles, we deform the contour into the the unphysical domain shown in Fig. 4(b).

The contour in Fig. 4(b) picks up contributions from all the bound and resonant poles so that the Green's function can be written by a simple sum formation as the Mittag-Leffler expansion [15-17],

$$
\mathcal{G}(q)=\sum_{n} \frac{\left|\phi_{n}\right\rangle\left\langle\tilde{\phi}_{n}\right|}{q-q_{n}}
$$

(The Mittag-Leffler expansion of the Green's function at finite temperature is discussed in Ref. [18]).

The advantage of this representation, Eq. (13), is that each component in the series is explicitly written in a simple form by the residue and position of the pole. Now by imposing the

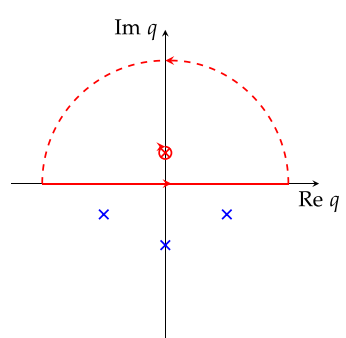

(a)

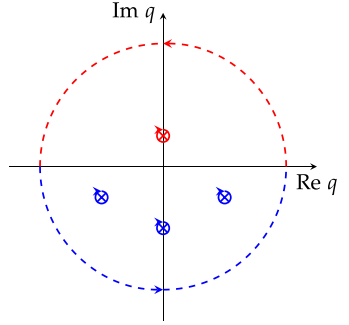

(b)
FIG. 4. Schematic of the poles and contours on the $q$ plane. The contour of Fig. 4(a) corresponds to the spectral representation, Eq. (9) (Fig. 3). By deforming the contour as Fig. 4(b), we obtain the Mittag-Leffler expansion, Eq. (13).

symmetry condition onto Eq. (13), we can write it in the form of the sum of contributions from each pair as

$$
\mathcal{G}(q)=\sum_{n} \mathcal{A}_{n}(q)=\sum_{n}\left(\frac{c_{n}}{q-q_{n}}-\frac{c_{n}^{*}}{q+q_{n}^{*}}\right),
$$

where $n$ denotes each pair. One notable point of Eq. (14) is that by imposing the pole symmetry properties, each pair contribution naturally behaves in the proper way at the thresholds, except for the positive definite property. The positive definiteness is satisfied by taking the sum of all pairs. Fundamentally, the pole symmetries and the threshold behaviors both originate from the same condition: the unitarity of the $S$ matrix.

The imaginary part of $A_{n}$ can be written as

$$
\operatorname{Im} \mathcal{A}_{n}(q)=\operatorname{Im} \frac{c_{n}\left(q+q^{*}\right)}{|q|^{2}+q_{n}\left(q-q^{*}\right)-q_{n}^{2}} .
$$

Under the threshold, $q$ is purely imaginary, and therefore, the contribution from a pair is identically 0 . Right above the threshold, the pair contributes linearly in terms of $q$. Thus in the vicinity of $\sqrt{s}=\varepsilon$, the imaginary part of $\mathcal{A}_{n}$ can be written as

$$
\operatorname{Im} \mathcal{A}_{n}(q)= \begin{cases}0 & (\sqrt{s}<\varepsilon) \\ -\operatorname{Im} \frac{2 c_{n}}{q_{n}^{2}} q+O\left(q^{2}\right) & (\sqrt{s}>\varepsilon) .\end{cases}
$$

Equation (16) coincides with Eq. (3).

The spectral representation, Eq. (9), is nothing but the unsubtracted dispersion relation and the integral may not always converge. In this case, one should replace Eq. (9) with the corresponding once-subtracted dispersion relation. Then the Mittag-Leffler expansion differs from Eq. (13) by a constant [originating from the outer circle in the contour in Fig. 4(b)]. By imposing the unitarity condition, however, the subtraction constant of the amplitude is fixed real. Thus the imaginary part of the amplitude will have no such ambiguity and will be the same as the imaginary part of Eq. (14).

In the case of a double-channel system, the Green's function has two branches with branch points at threshold energies squared, $\varepsilon_{1}^{2}$ and $\varepsilon_{2}^{2}$, as shown in Fig. 5. To express the Green's function in the form of the Mittag-Leffler expansion, one must choose an appropriate variable and unfold the four Riemann sheets $[3,14]$. The basic strategy is as follows. By the change 


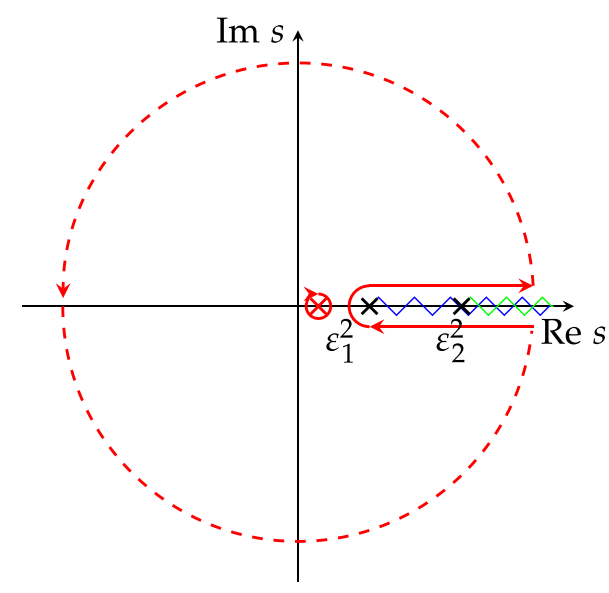

FIG. 5. The contour corresponding to the spectral representation, Eq. (9), in the case of a double-channel system. The contour detours two branch cuts that run along the real axis from each threshold, $\varepsilon_{1}^{2}$ (blue) and $\varepsilon_{2}^{2}$ (green), to $\infty$.

of variables, we send one of the branch points to infinity. Then the structure of the Riemann surface becomes the same as the single-channel case, which we can easily unfold. The appropriate variable $z$ is given by

$$
z=\frac{1+\sqrt{u}}{1-\sqrt{u}}, \quad u=\frac{q_{1}-\Delta}{q_{1}+\Delta},
$$

where $q_{i}=\sqrt{s-\varepsilon_{i}^{2}}=\sqrt{\frac{\varepsilon_{i}}{\mu_{i}}} k_{i}+O\left(k_{i}^{3}\right)$ and $\Delta=\sqrt{\varepsilon_{2}^{2}-\varepsilon_{1}^{2}}$.

By the same argument as in the single-channel case, the Green's function can be written in the variable $z$ as

$$
\mathcal{G}(z)=\sum_{n} \mathcal{A}_{n}(z)=\sum_{n}\left(\frac{c_{n}}{z-z_{n}}-\frac{c_{n}^{*}}{z+z_{n}^{*}}\right) .
$$

We call Eq. (18), the uniformized Mittag-Leffler expansion (Fig. 6). Note that $z \rightarrow-z^{*}$ when $q_{1} \rightarrow-q_{1}^{*}$ and $q_{2} \rightarrow-q_{2}^{*}$. Thus, the same symmetric conditions hold for the poles in the $z$ plane:

$$
\mathcal{S}^{*}\left(-z^{*}\right)=\mathcal{S}(z)
$$

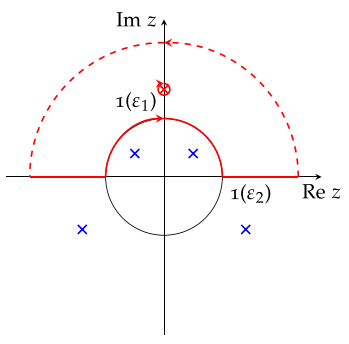

(a)

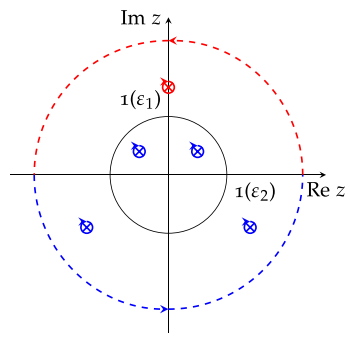

(b)
FIG. 6. Schematic of the poles and contours on the $z$ plane. The contour in (a) corresponds to the spectral representation, Eq. (9) (Fig. 3). By deforming the contour as in (b), we obtain the uniformized Mittag-Leffler expansion.
The threshold behaviors are given in the vicinity of $\sqrt{s}=\varepsilon_{1}$ as

$$
\operatorname{Im} \mathcal{A}_{n}(z)= \begin{cases}0 & \left(\sqrt{s}<\varepsilon_{1}\right) \\ -\operatorname{Im} \frac{2 c_{n}}{\left(z_{n}-i\right)^{2}} \frac{q_{1}}{\Delta}+O\left(q_{1}^{2}\right) & \left(\sqrt{s}>\varepsilon_{1}\right)\end{cases}
$$

and in the vicinity of $\sqrt{s}=\varepsilon_{2}$ as

$$
\begin{aligned}
& \operatorname{Im} \mathcal{A}_{n}(z) \\
& =\left\{\begin{array}{l}
\operatorname{Im} \frac{2 c_{n}}{1-z_{n}^{2}}-\operatorname{Re} \frac{4 c_{n} z_{n}}{\left(1-z_{n}^{2}\right)^{2}} \frac{\tilde{q}_{2}}{\Delta}+O\left(\tilde{q}_{2}^{2}\right) \quad\left(\sqrt{s}<\varepsilon_{2}\right), \\
\operatorname{Im} \frac{2 c_{n}}{1-z_{n}^{2}}-\operatorname{Im} \frac{2 c_{n}\left(1+z_{n}^{2}\right)}{\left(1-z_{n}^{2}\right)^{2}} \frac{q_{2}}{\Delta}+O\left(q_{2}^{2}\right) \quad\left(\sqrt{s}>\varepsilon_{2}\right),
\end{array}\right.
\end{aligned}
$$

where $\tilde{q_{2}}$ is defined by $q_{2}=i \tilde{q}_{2}$. Equations (20) and (21) coincide with Eqs. (3) and (4), respectively.

For systems with three or more channels, the Riemann surface of the Green's function cannot be uniformized into a single complex plane. For example, the Riemann surface of a three-channel system is topologically equivalent to a torus [3]. In these cases, there is no simple variable to express the Green's function in the form of Eq. (13). Nevertheless, one can unfold a local region which may be sufficient when considering a particular energy region.

To summarize, by appropriately uniformizing the Riemann surface, we can expand the Green's function or $T$ matrix by the uniformized Mittag-Leffler expansion. The symmetry conditions on the poles naturally lead to the proper threshold behaviors.

\section{NEW PROPOSAL FOR DETERMINATION OF COMPLEX RESONANCE ENERGIES}

Based on the discussion above that observables can be expressed as the imaginary part of the sum of all pole terms in the uniformized complex plane, we propose the following procedures to extract information on the complex energies and residues of the resonance poles from observables in a model-independent manner.

i. Find an appropriate kinetic variable, $z$, that uniformizes the system.

ii. Assume that the amplitude, $\mathcal{A}(z)$, whose imaginary part gives observables such as the cross section, $\sigma$, and the invariant or missing-mass distribution, $\mathcal{N}$, is approximated by a few $(m)$ pairs of the pole terms as

$$
\mathcal{A}(z)=\sum_{n=1}^{m}\left(\frac{c_{n}}{z-z_{n}}-\frac{c_{n}^{*}}{z+z_{n}^{*}}\right) .
$$

iii. Determine the complex pole positions and residues, $z_{n}$ and $c_{n},(n=1, \ldots, m)$, by fitting $\operatorname{Im} \mathcal{A}(z)$ to the experimental data, from which the complex energies and residues of resonances are obtained.

Here, we clarify the reliability of the obtained results. If one increases the number of pairs of poles in the sum, the positions of the complex poles and residues, $z_{n}$ and $c_{n}$, would change in general. If the fitting is successful, then $z_{n}$ and $c_{n}$ 


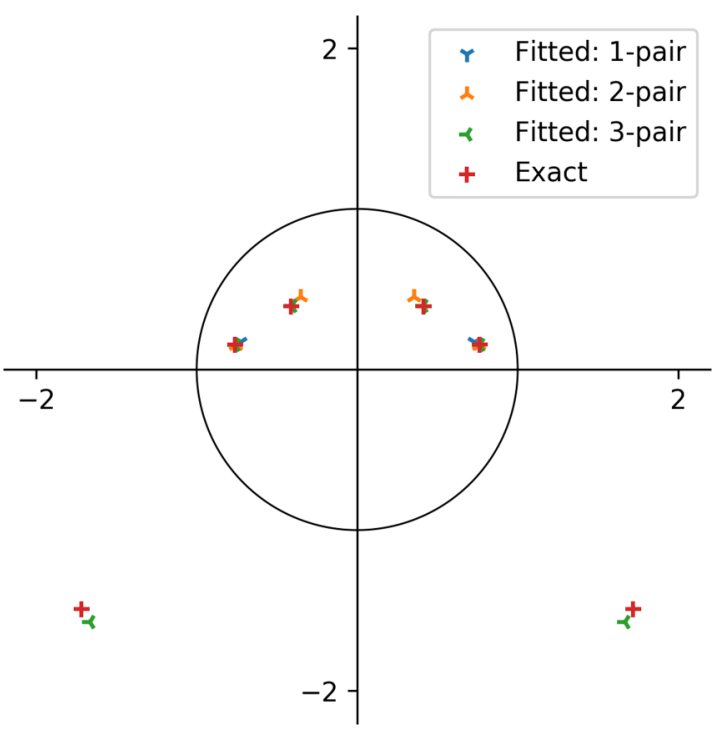

FIG. 7. The fitted pole positions of $\operatorname{Im} \mathcal{T}$ on the $z$ plane when considering one, two, and three resonant pairs $(m=1,2,3)$. Note that the fitted pole positions in the upper half-plane almost overlap the exact positions in the case of $m=2,3$ for the pole near $z=1(\bar{K} N$ threshold) and $m=3$ for the pole closer to $z=i$ ( $\pi \Sigma$ threshold).

would change little for the poles near the fitting energy region, while they might change to some extent for the poles away from the fitting energy region. Besides, the position of the newly added pair should be most away from the fitting energy region. Ideally, if this condition is met, one could conclude that the fitting is successful, and the obtained complex positions and residues for the poles near the fitting energy region are regarded as reliable results.

The counterpart of the resonance pole may be very far from the physical region of interest. Then its contribution is negligible, and the contribution of the resonance pole alone gives the threshold behavior in practice. Nevertheless, the inclusion of the resonance pole's counterpart does not increase the number of parameters and, therefore, does nothing wrong.

Also, note that the method is model independent in the sense that the procedure does not depend on a particular model. The only necessary information is the channels of the relevant particles.

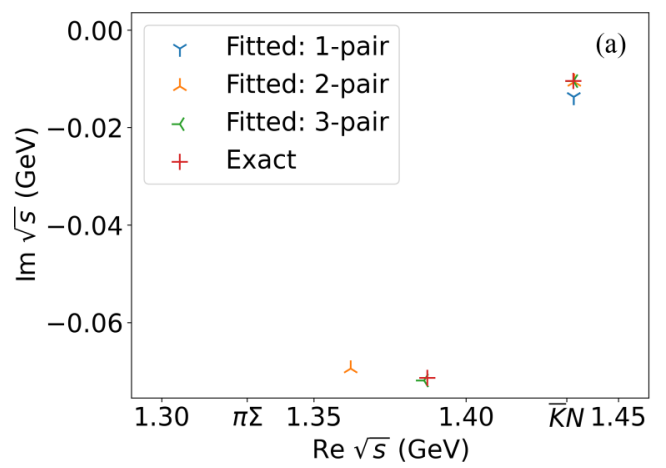

\section{RESULTS WITH MODEL THEORY}

Here we apply the uniformized Mittag-Leffler expansion to a model theory and compare the results, pole positions and residues, to the exact model-calculated results. For the model theory, we consider a meson-baryon scattering in a chiralunitary model [19-22] involving two channels, $\bar{K} N(I=0)$ and $\pi \Sigma(I=0)$. Since the system is a double-channel system, the appropriate kinetic variable is given by $z$ in Eq. (17). In addition to the resonant poles, there is a pole at $s=0$ (corresponding to two poles $z_{ \pm}$on the $z$ plane) originating from relativistic kinematics. Since the $s=0$ poles are of kinematic origin, they do not depend on the interaction strength of the model,

$$
z_{ \pm}=\frac{\sqrt{ \pm i \varepsilon_{1}+\Delta}+\sqrt{ \pm i \varepsilon_{1}-\Delta}}{\sqrt{ \pm i \varepsilon_{1}+\Delta}-\sqrt{ \pm i \varepsilon_{1}-\Delta}}
$$

where, $\varepsilon_{1}=m_{\pi}+m_{\Sigma}, \varepsilon_{2}=m_{\bar{K}}+m_{N}$, and $\Delta=\sqrt{\varepsilon_{2}^{2}-\varepsilon_{1}^{2}}$. Details about the numerical calculation of the model and the $s=0$ poles are discussed in the Appendix.

The fitted results are obtained by applying the method in Sec. III, regarding the numerical model results as virtual experimental data. We approximated the model-calculated $T$ matrix by the Mittag-Leffler expansion considering the contributions from $m$-resonant pairs and the $s=0$ poles as

$$
\operatorname{Im} \mathcal{T}(z)=\sum_{n=1}^{m}\left(\frac{c_{n}}{z-z_{n}}-\frac{c_{n}^{*}}{z+z_{n}^{*}}\right)+\sum_{ \pm} \frac{d_{ \pm}}{z-z_{ \pm}},
$$

where the fitting parameters are $c_{n}, z_{n}$, and $d_{ \pm}$( $d_{ \pm}$is purely imaginary). We have four real parameters for each pair of resonance poles- two for the pole position and two for the residue - and one for the residue of each kinematical pole at $z_{ \pm}$. In the present case, the number of resonant poles in the neighborhood of the thresholds is known from the model calculation. There are three resonant pole pairs in the vicinity of the two thresholds: two pairs on the $(-+)$ sheet of the $\sqrt{s}$ plane and one pair on the $(--)$ sheet. (The four Riemann sheets of the $\sqrt{s}$ plane can be labeled by the $\operatorname{sign}$ of $\operatorname{Im} q_{i}$. We label the sheets $\left(\operatorname{sgn}\left(\operatorname{Im} q_{1}\right)\right.$ and $\operatorname{sgn}\left(\operatorname{Im} q_{2}\right)$ [7]). When fitting actual experimental data, however, the number of poles

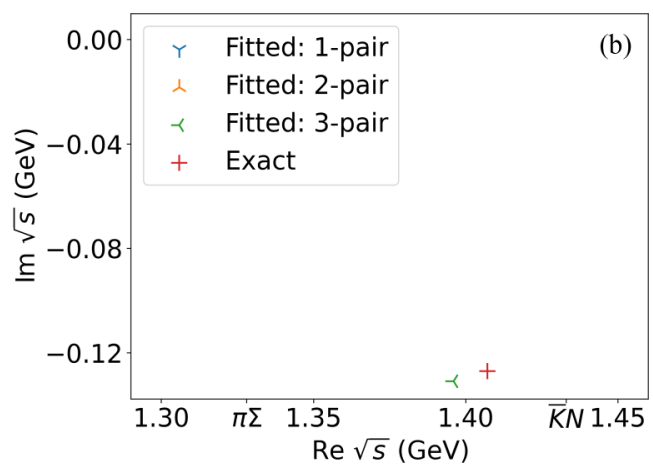

FIG. 8. The fitted pole positions of $\operatorname{Im} \mathcal{T}$ on the $\sqrt{s}$ plane when considering one, two, and three resonant pairs $(m=1,2,3)$. (a) The $(-+)$ sheet; (b) the (--) sheet. The $\pi \Sigma$ and $\bar{K} N$ labels on the real $\sqrt{s}$ axis represent their threshold energies. Note that the fitted pole positions in the (-+) sheet almost overlap the exact positions in the case of $m=2,3$ for the pole near the $\bar{K} N$ threshold and $m=3$ for the pole closer to the $\pi \Sigma$ threshold. 
TABLE I. Fitted pole positions of $\operatorname{Im} \mathcal{T}$ for the case of $m=1,2,3$, and the exact pole positions from the model calculation. The upper rows list the positions on the $z$ plane (dimensionless), and the lower rows the positions on the $\sqrt{s}$ plane (units of $\mathrm{GeV}$ ).

\begin{tabular}{lcccc}
\hline \hline & $m=1$ & $m=2$ & $m=3$ & Exact \\
\hline Pole: $1,1^{*}$ & $\pm 0.731+0.173 i$ & $\pm 0.756+0.153 i$ & $\pm 0.760+0.154 i$ & $\pm 0.760+0.154 i$ \\
& $1.435 \mp 0.014 i$ & $1.436 \mp 0.010 i$ & $1.435 \mp 0.010 i$ & $1.435 \mp 0.010 i$ \\
Pole: $2,2^{*}$ & & $\pm 0.355+0.453 i$ & $\pm 0.409+0.397 i$ & $\pm 0.413+0.395 i$ \\
& & $1.362 \mp 0.069 i$ & $1.386 \mp 0.072 i$ & $1.387 \mp 0.071 i$ \\
Pole: $3,3^{*}$ & & $\pm 1.67-1.57 i$ & $1.72-1.49 i$ \\
& & & $1.396 \mp 0.131 i$ & $1.407 \mp 0.127 i$ \\
\hline \hline
\end{tabular}

that need consideration is unknown. Here, we start by fitting the exact $T$ matrix in the case of $m=1$ and then increase the number of resonant pairs considered. As we do so, we presume that the positions of the preexisting pairs will move toward the exact results, and a new pair is found farther away from the region of interest. Then, at some point in the process, all poles in the vicinity of the thresholds would converge and we can regard these fitted results as the actual pole positions.

Figures 7 and 8 and Table I show the fitted positions of the resonant poles of the $T$ matrix when considering one, two, and three resonant pairs, respectively. As expected from the discussion in the previous paragraph, the fitted pole positions converge to the exact model-calculated results as we increase the number of resonant pairs from one to three. When we increase the number of resonant pairs to four, however, an overfitting occurs. For now, let us postpone the discussion of overfitting and consider the case of fitting $\operatorname{Im} \mathcal{T}$ with three resonant pairs in more detail.

The fitted results of the imaginary part of the $T$ matrix are given in Figs. 9-11 and Tables II and III. In Figs. 9-11,

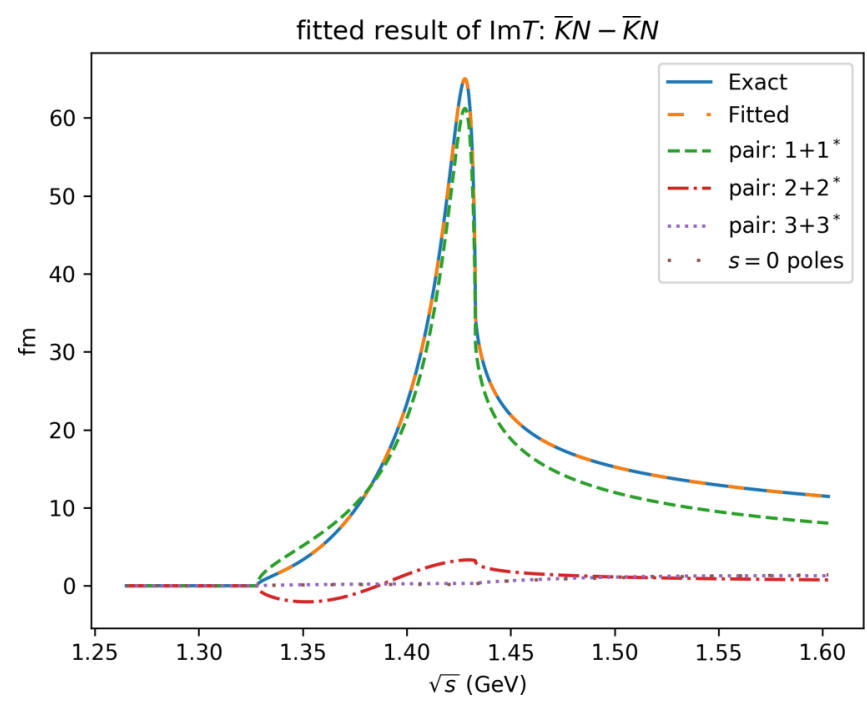

FIG. 9. Exact model results and fitted results of $\operatorname{Im} \mathcal{T}$ for the $\bar{K} N-\bar{K} N$ component. We also show the contributions from each pair of poles $1+1^{*}, 2+2^{*}$, and $3+3^{*}$ and the $s=0$ poles for the fitted $\operatorname{Im} \mathcal{T}$. The fitted results are indistinguishable from the exact model results, exhibiting the validity of our method. The contributions from the $s=0$ poles are insignificant. The specifications are given. we plot and compare the fitted uniformized Mittag-Leffler expansion [Eq. (24)] to the results of the exactly calculated $\operatorname{Im} \mathcal{T}$, in the case of $m=3$. The difference between the fitted results and the exact model results is hardly distinguishable. Also shown in Figs. 9-11 are the contributions from each pair of poles and the $s=0$ poles. The contribution of the $1+1^{*}$ pair explains most of the $\bar{K} N-\bar{K} N$ component. For the $\pi \Sigma-\bar{K} N$ component, the contribution of the $1+1^{*}$ pair is still the largest, but that of $2+2^{*}$ is also considerable. For the $\pi \Sigma-\pi \Sigma$ component, the contributions of both $1+1^{*}$ and $2+2^{*}$ are essential, but the latter is larger than the former. All $1+1^{*}, 2+2^{*}$, and $3+3^{*}$ pairs contribute above the $\bar{K} N$ threshold for the $\pi \Sigma-\bar{K} N$ and $\pi \Sigma-\pi \Sigma$ components. The contribution of $s=0$ poles is insignificant everywhere in the range of interest. Thus, even if we do not consider the contribution from the $s=0$ poles, we expect that the fitted results should hardly change. From the results above, we can conclude that the poles $1+1^{*}$ and $2+2^{*}$ explain the peak structures and $3+3^{*}$ gives the background contributions of the virtual experimental data.

In Tables II, and III, we show the fitted results of the pole positions and residues of the $T$ matrix in the $z$ plane and $\sqrt{s}$ plane, respectively. The exactly calculated results in the model theory are also shown for comparison for poles 1, 2, and 3. From these results, combined with Figs. 7 and 8, we can acknowledge that the fitted positions and residues of poles

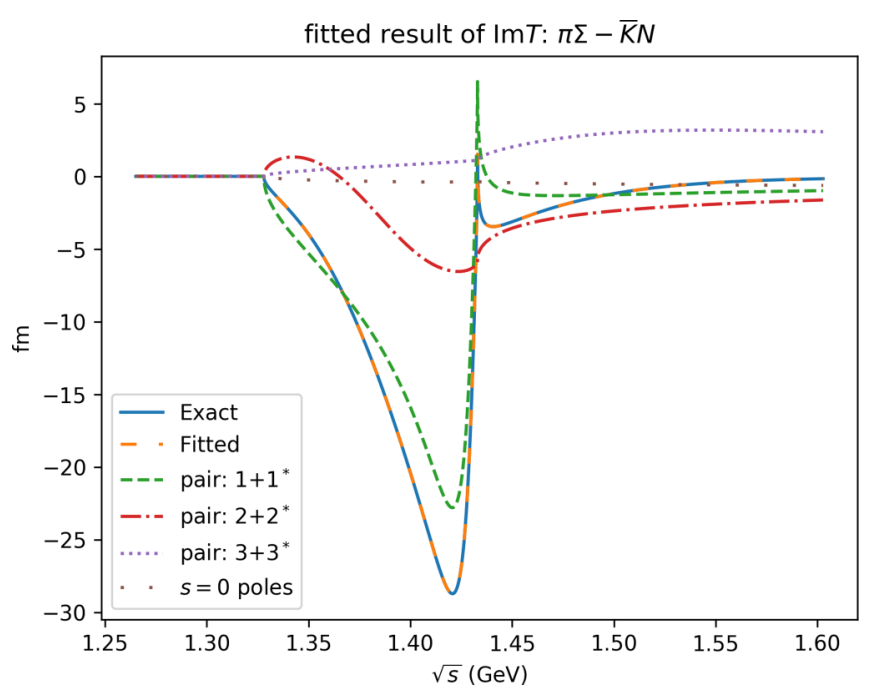

FIG. 10. The same as Fig. 9, for the $\pi \Sigma-\bar{K} N$ component. 


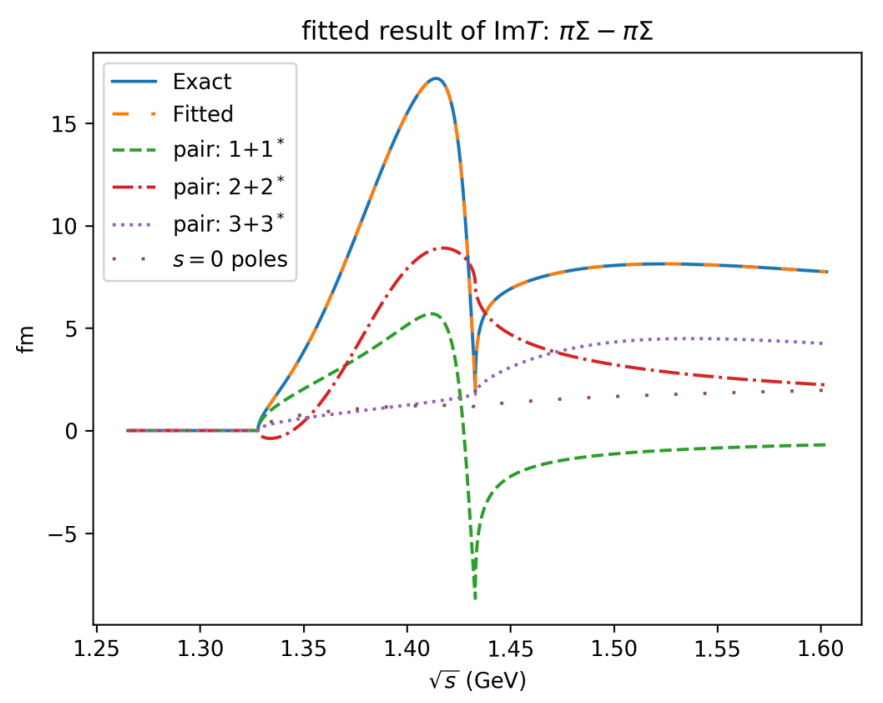

FIG. 11. The same as Fig. 9, for the $\pi \Sigma-\pi \Sigma$ component.

agree very well with those of the exact calculation. Pole $1\left(1^{*}\right)$ is located close to the real $\sqrt{s}$ axis, and the difference between the fitted and the exact results is minimal. As the distance from the real $\sqrt{s}$ axis increases, for pole $2\left(2^{*}\right)$ and further for pole $3\left(3^{*}\right)$, the difference between the fitted and the exact results widens. However, even for pole $3\left(3^{*}\right)$, the difference is still rather small. We also note that the difference seems to increase when moving from the complex $z$ plane to the complex $\sqrt{s}$ plane.

As discussed in a prior paragraph, as we increase the number of resonant pairs to four, an overfitting occurs (Fig. 12). Instead of locating a new pole farther from the threshold, it accumulates near the third resonant pair. The overfitting is an artifactual phenomenon due to the fitting procedure where the contribution from the third resonant pair will split into two so that the contribution from the first and second terms amount to the contribution of the original term. Most likely, one has to extend the fitting range to obtain a reasonable behavior of the fourth pair. Thus, in the present case, we decide that it is sufficient to consider three resonant-pair contributions, which is inevitably the same as the number of resonant pairs in the thresholds' vicinity in the model calculation.

To summarize, the imaginary part of the model-calculated $T$ matrix can be expressed extremely well with the uniformized Mittag-Leffler expansion when considering three
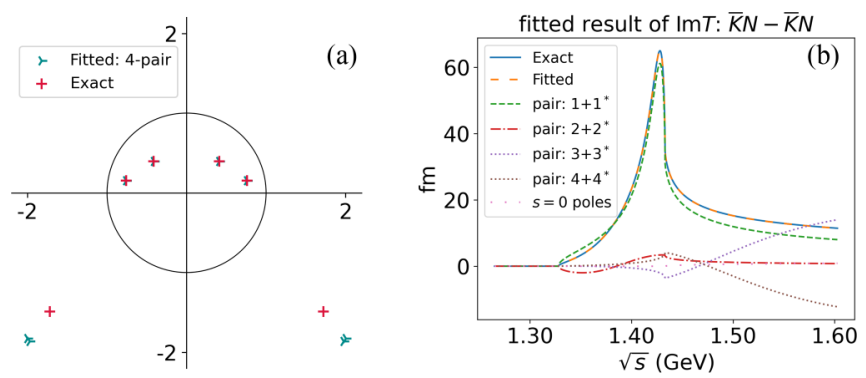

FIG. 12. Overfitting when considering four resonant-pair contributions. The fourth resonant pair accumulates near the third pair, and its contribution cancels the third contribution, which amounts to the original third contribution when considering three resonant pairs.

resonant pairs. The fitted poles and residues are in good agreement with the exact model calculations.

\section{CONCLUSION}

From the results in Sec. IV, we conclude that if experimentalists can provide us with data of sufficient quality and quantity, we can perfectly reproduce the experimental data and determine the complex energies and the residues in a completely model-independent manner. The method accurately reproduces not only the peak structures but also continuous spectra with proper threshold behaviors, in a natural way. Realistically, however, the experimental circumstances may not be perfect. Even in such a situation, the use of the uniformized Mittag-Leffler expansion would provide us with a framework which is theoretically more reasonable and practically more useful than the usual methods in the sense that it automatically incorporates the proper threshold behaviors.

\section{ACKNOWLEDGMENTS}

The authors would like to thank Kazuki Yamada for the discussion in the early stage of the work. O.M. would also like to thank Toru Sato, Koichi Yazaki, and the members of the discussion meeting held at KEK Tokai campus, Yoshinori Akaishi, Akinobu Dote, Toru Harada, Fuminori Sakuma, and Shoji Shinmura.

\section{APPENDIX: DETAILS OF THE MODEL THEORY}

In the model calculation in Sec. IV, we calculated the $T$ matrix of a two-channel system involving channels of

TABLE II. Fitted results of the pole positions and residues of $i \mathcal{T}$ in the $z$ plane. Values in parentheses are the exact model results. Pole positions are dimensionless and residues have units of $\mathrm{fm}$.

\begin{tabular}{lcccc}
\hline \hline & Pole position $(z)$ & $\pi \Sigma-\pi \Sigma$ & $\pi \Sigma-\bar{K} N$ & $\bar{K} N-\bar{K} N$ \\
\hline Pole: $1,1^{*}$ & $\pm 0.760+0.154 i$ & $-0.901 \pm 2.72 i$ & $-1.56 \mp 6.52 i$ & $11.4 \pm 4.79 i$ \\
& $( \pm 0.760+0.154 i)$ & $(-0.892 \pm 2.72 i)$ & $(-1.57 \mp 6.51 i)$ & $(11.4 \pm 4.78 i)$ \\
Pole: $2,2^{*}$ & $\pm 0.409+0.397 i$ & $3.27 \mp 1.99 i$ & $-2.35 \pm 2.41$ & $1.05 \mp 2.10 i$ \\
& $( \pm 0.413+0.395 i)$ & $(3.31 \mp 1.85 i)$ & $(-2.41 \pm 2.34 i)$ & $(1.11 \mp 2.06 i)$ \\
Pole: $3,3^{*}$ & $\pm 1.67-1.57 i$ & $6.00 \pm 3.94 i$ & $4.67 \pm 2.31 i$ & $(1.95 \pm 0.814 i)$ \\
& $( \pm 1.72-1.49 i)$ & $(4.83 \pm 3.92 i)$ & $(3.70 \pm 2.30 i)$ & $0.461 i$ \\
\hline \hline
\end{tabular}




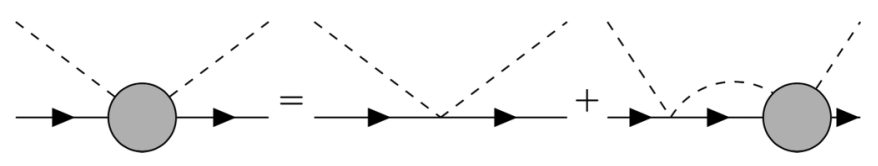

FIG. 13. Ladder diagrams taken into account when calculating the amplitude. The circle represents the complete meson-baryon vertex. Dashed lines and solid lines with arrows represent the meson and baryon propagators, respectively.

$\bar{K} N(I=0)$ and $\pi \Sigma(I=0)$ using the chiral-unitary model. The diagrams taken into consideration are shown in Fig. 13 and the interaction is given by the lowest-order meson-baryon chiral Lagrangian,

$$
\mathcal{L}_{\mathrm{TW}}=i \frac{C_{i j}}{4 f^{2}}\left(\bar{\psi} \phi^{\dagger}\right)_{j} \overleftrightarrow{\not \partial}(\phi \psi)_{i},
$$

where $i$ and $j$ denote the channels of the particles (in this case $\pi \Sigma$ or $\bar{K} N$ ) and $C_{i j}$ are the Clebsh-Gordan coefficients. For the channels of interest, $C_{i j}$ is given by

$$
C_{i j}=\left(\begin{array}{cc}
3 & -\sqrt{\frac{3}{2}} \\
-\sqrt{\frac{3}{2}} & 4
\end{array}\right),
$$

where $i,(j)=1$ corresponds to $\bar{K} N(I=0)$, and $i,(j)=2$ corresponds to $\pi \Sigma(I=0)$.

The basic outline of the calculation follows Ref. [22]. In this paper, however, we have replaced the Klein-Gordon prop- agators of the baryons with Dirac propagators. Thus, minor changes were made in the calculations.

The $T$ matrix of the scattering of channel $i \rightarrow j$ is given by

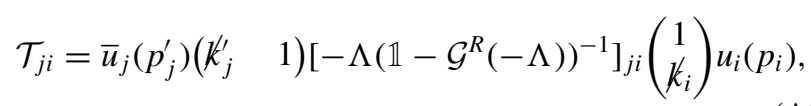

where $\Lambda$ and $\mathcal{G}^{R}$ are $4 \times 4$ matrices, and the $u(p)$ 's are free Dirac spinors. $\Lambda$ is the matrix which characterizes the strength of the coupling between channels, and $\mathcal{G}^{R}$ is the renormalized one-loop contribution,

$$
\Lambda_{j i}=\frac{C_{j i}}{4 f^{2}} \mathbb{1}_{2 \times 2}, \quad \mathcal{G}_{j i}^{R}=\delta_{j i} \mathcal{G}_{i}^{R}
$$

Here we denote the components of $\mathcal{G}_{i}^{R}$ as

$$
\mathcal{G}_{i}^{R}=\left(\begin{array}{ll}
\mathcal{G}_{1 i}^{R} & \mathcal{G}_{0 i}^{R} \\
\mathcal{G}_{2 i}^{R} & \mathcal{G}_{1 i}^{R}
\end{array}\right)
$$

The $\mathcal{G}$ 's are related to the one-loop contributions with KleinGordon propagators, $G$ 's, as

$$
\begin{aligned}
& \mathcal{G}_{0 i}^{R}=\left(\not P+M_{i}\right) G_{0 i}^{R}-G_{1 i}^{R} \not P, \\
& \mathcal{G}_{1 i}^{R}=\left(\not P+M_{i}\right) G_{1 i}^{R} \not P-G_{2 i}^{R}, \\
& \mathcal{G}_{2 i}^{R}=\left(P^{2}-M_{i}^{2}\right) G_{1 i}^{R} \not P-\left(\not P-M_{i}\right) G_{2 i}^{R},
\end{aligned}
$$

where $P$ is the total momentum and $M_{i}$ is the mass of the baryon in channel $i$. The $G$ 's are explicitly given by

$$
\begin{aligned}
G_{0 i}^{R}(\sqrt{s})= & \frac{2 M_{i}}{16 \pi^{2}}\left\{a_{0}^{i}(\mu)+\log \frac{M_{i}^{2}}{\mu^{2}}+\frac{m_{i}^{2}-M_{i}^{2}+s}{2 s} \log \frac{m_{i}^{2}}{M_{i}^{2}}+\frac{\bar{q}_{i}}{\sqrt{s}} \log \frac{\phi_{++}^{i} \phi_{+-}^{i}}{\phi_{-+}^{i} \phi_{--}^{i}}\right\}, \\
G_{1 i}^{R}(\sqrt{s})= & \frac{M_{i}}{16 \pi^{2}}\left\{a_{1}^{i}(\mu)+\log \frac{M_{i}^{2}}{\mu^{2}}+\frac{M_{i}^{2}-m_{i}^{2}}{s}+\frac{\left(m_{i}^{2}-M_{i}^{2}+s\right)^{2}+4 s \bar{q}_{i}^{2}}{4 s^{2}} \log \frac{m_{i}^{2}}{M_{i}^{2}}-\frac{\bar{q}_{i}}{\sqrt{s}} \frac{m_{i}^{2}-M_{i}^{2}+s}{2 s} \log \frac{\phi_{++}^{i} \phi_{+-}^{i}}{\phi_{-+}^{i} \phi_{--}^{i}}\right\}, \\
G_{2 i}^{R}(\sqrt{s})= & \frac{2 M_{i}}{16 \pi^{2}}\left\{\left(M_{i}^{2}+m_{i}^{2}\right)\left(a_{2}^{i}(\mu)+\log \frac{M_{i}^{2}}{\mu^{2}}\right)+\frac{s}{2}-m_{i}^{2}-2 \bar{q}_{i}^{2}+\frac{\left(M_{i}^{2}-m_{i}^{2}\right)^{2}}{2 s}\right. \\
& \left.-\frac{\left(m_{i}^{2}-M_{i}^{2}+s\right)\left[\left(m_{i}^{2}-M_{i}^{2}+s\right)^{2}-4 s\left(\bar{q}_{i}^{2}+2 m_{i}^{2}\right)\right]}{8 s^{2}} \log \frac{m_{i}^{2}}{M_{i}^{2}}-\frac{\bar{q}_{i}}{\sqrt{s}} \frac{\left(m_{i}^{2}-M_{i}^{2}+s\right)^{2}-4 s\left(\bar{q}_{i}^{2}+2 m_{i}^{2}\right)}{4 s} \log \frac{\phi_{++}^{i} \phi_{+-}^{i}}{\phi_{-+}^{i} \phi_{--}^{i}}\right\},
\end{aligned}
$$

where $s=P^{2}, \bar{q}_{i}=\sqrt{\left(s-\left(M_{i}+m_{i}\right)^{2}\right)\left(s-\left(M_{i}-m_{i}\right)^{2}\right) / 4 s}$, and $\phi_{ \pm \pm}^{i}= \pm s \pm\left(M_{i}^{2}-m_{i}^{2}\right)+2 \bar{q}_{i} \sqrt{s} . M_{i}$ and $m_{i}$ are the baryon and meson masses in channel $i$, respectively. $a_{0}^{i}, a_{1}^{i}$, and $a_{2}^{i}$ are the renormalization constants which are determined by the renormalization condition.

TABLE III. Fitted results of the pole positions and residues of $i \mathcal{T}$ in the $\sqrt{s}$ plane. Values in parentheses are the exact model results. Pole positions and residues have units of $\mathrm{GeV}$ and $\mathrm{fm} / \mathrm{GeV}$, respectively.

\begin{tabular}{lcccc}
\hline \hline & Pole position $(\sqrt{s})$ & $\pi \Sigma-\pi \Sigma$ & $\pi \Sigma-\bar{K} N$ & $\bar{K} N-\bar{K} N$ \\
\hline Pole: $1,1^{*}$ & $1.435 \mp 0.010 i$ & $\pm 31.7-10.7 i$ & $\mp 49.9+60.2 i$ & $(\mp 35.2-140 . i$ \\
& $(1.435 \mp 0.010 i)$ & $( \pm 31.6-10.7 i)$ & $(\mp 49.7+60.2 i)$ & $(\mp 35.3-140 . i)$ \\
Pole: $2,2^{*}$ & $1.386 \mp 0.072 i$ & $\pm 1.81-12.1 i$ & $\pm 1.12+10.7 i$ & $( \pm 1.03+10.8 i)$ \\
& $(1.387 \mp 0.071 i)$ & $( \pm 2.03-12.1 i)$ & $\pm 18.3+38.3 i$ & $(\mp 2.95-6.907 i)$ \\
Pole: $3,3^{*}$ & $1.397 \mp 0.131 i$ & $\pm 18.7+55.4 i$ & $( \pm 13.4+33.5 i)$ & $( \pm 9.01+15.0 i)$ \\
& $(1.407 \mp 0.127 i)$ & $( \pm 13.0+49.8 i)$ & &
\end{tabular}


TABLE IV. Parameters used in the model calculation. The baryon and meson masses, $M$ and $m$, the pion decay constant, $f$, and the dimensional regularization parameter, $\mu$, are given in units of GeV. The renormalization parameters, $a^{\bar{K} N}$ and $a^{\pi \Sigma}$, are dimensionless and satisfy the renormalization condition, Eq. (A5).

\begin{tabular}{lcccccccccc}
\hline \hline$m_{\bar{K}}$ & $m_{\pi}$ & $M_{N}$ & $M_{\Sigma}$ & $f$ & $\mu$ & $a_{0}^{\bar{K} N}$ & $a_{1}^{\bar{K} N}$ & $a_{2}^{\bar{K} N}$ & $a_{0}^{\pi \Sigma}$ & $a_{1}^{\pi \Sigma}$ \\
\hline 0.495 & 0.135 & 0.938 & 1.193 & 0.10377 & 0.630 & -1.87 & -1.66 & -1.41 & -1.95 & -1.86 \\
\hline \hline
\end{tabular}

By definition, $\bar{q}_{i}$ behaves as $O\left(\frac{1}{\sqrt{s}}\right)$ as $s$ approaches 0 . Observing the explicit form of the $G$ 's given by Eq. (A3), the factors of $q_{i}$ are always accompanied by a factor of $\sqrt{s}$ or $\frac{1}{\sqrt{s}}$, and therefore the $G$ 's will have a pole-type singularity at $s=0$. The $G$ 's only depend on the structure of the propagators and are totally kinematic (all the information on the interaction is contained in matrix $\Lambda$ ). Thus the $T$ matrix will have poles at $s=0$ that are of kinematic origin, which does not depend on the interaction strength.

The $T$ matrix can be expanded in powers of $\sqrt{s}-M$ as

$$
\begin{aligned}
\mathcal{T}_{j i}= & -\bar{u}_{j}\left(p_{j}^{\prime}\right)\left(\begin{array}{ll}
k_{j}^{\prime} & 1
\end{array}\right) \Lambda_{j i}\left(\begin{array}{c}
1 \\
k_{i}
\end{array}\right) u_{i}\left(p_{i}\right)+\bar{u}_{j}\left(p_{j}^{\prime}\right) \Lambda_{j k}\left[3\left(M_{k} G_{1 k}^{R}-\frac{G_{2 k}^{R}}{2 M_{k}}\right)\left(\sqrt{s}-M_{k}\right)\right. \\
& \left.+\left(4 G_{1 k}^{R}+G_{0 k}^{R}\right)\left(\sqrt{s}-M_{k}\right)^{2}+\frac{G_{1 k}^{R}}{2 M_{k}}\left(\sqrt{s}-M_{k}\right)^{3}\right] \Lambda_{k i} u_{i}\left(p_{i}\right)+O(\sqrt{s}-M)^{2}
\end{aligned}
$$

where the first and second terms correspond to the tree and one-loop contributions, respectively. Here we set the renormalization condition so that at the lowest order of $\sqrt{s}-M$, the $\mathcal{T}_{j i}$ is the same as the tree contribution, meaning that the loop contributions should appear from the order of $O(\sqrt{s}-M)^{2}$. By setting the renormalization condition as mentioned, the renormalization parameters must satisfy the following equation:

$$
2 M_{i}^{2} G_{1 i}^{R}\left(\sqrt{s}=M_{i}\right)-G_{2 i}^{R}\left(\sqrt{s}=M_{i}\right)=0 .
$$

The parameters used in the numerical calculation are listed in Table IV.

[1] G. Breit and E. Wigner, Phys. Rev. 49, 519 (1936).

[2] L. Brown, Quantum Field Theory (Cambridge University Press, Cambridge, UK, 1992).

[3] R. G. Newton, Scattering Theory of Waves and Particles, 2nd ed. (Springer Science \& Business Media, New York, 2013).

[4] F.-K. Guo, C. Hanhart, Ulf-G. Meißner, Q. Wang, Q. Zhao, and B.-S. Zou, Rev. Mod. Phys. 90, 015004 (2018).

[5] M. Karliner, J. L. Rosner, and T. Skwarnicki, Annu. Rev. Nucl. Part. Sci. 68, 17 (2018).

[6] S. M. Flatte, Phys. Lett. B 63, 224 (1976).

[7] A. M. Badalian, L. P. Kok, M. I. Polikarpov, and Y. A. Simonov, Phys. Rep. 82, 31 (1982).

[8] R. H. Dalitz and S. F. Tuan, Ann. Phys. 10, 307 (1960).

[9] G. F. Chew and S. Mandelstam, Phys. Rev. 119, 467 (1960).

[10] A. L. Fetter and J. D. Walecka, Quantum Theory of ManyParticle Systems (McGraw-Hill, New York, 1971).
[11] G. Bertsch and S. Tsai, Phys. Rep. 18, 125 (1975).

[12] O. Morimatsu and K. Yazaki, Prog. Part. Nucl. Phys. 33, 679 (1994).

[13] M. M. Sternheim and J. F. Walker, Phys. Rev. C 6, 114 (1972).

[14] M. Kato, Ann. Phys. 31, 130 (1965).

[15] W. J. Romo, Nucl. Phys. A 237, 275 (1975).

[16] W. J. Romo, Nucl. Phys. A 302, 61 (1978).

[17] J. Bang, F. A. Gareev, M. Kh. Gizatkulov, S. A. Goncharov, Nucl. Phys. A 309, 381 (1978).

[18] Y. Hidaka, O. Morimatsu, T. Nishikawa, and M. Ohtani, Phys. Rev. D 68, 111901(R) (2003).

[19] N. Kaiser, P. B. Siegel, and W. Weise, Nucl. Phys. A 594, 325 (1995).

[20] E. Oset and A. Ramos, Nucl. Phys. A 635, 99 (1998).

[21] T. Hyodo and D. Jido, Prog. Part. Nucl. Phys. 67, 55 (2012).

[22] O. Morimatsu and K. Yamada, Phys. Rev. C 100, 025201 (2019). 